\title{
Hypermagnesemia and Spontaneous Ileal Perforation in a Preterm Infant
}

Karen Johal, MD, Shabih Manzar, MD

A case of a preterm infant with spontaneous ileal perforation is described in association with hypermagnesemia. No known causes (infection, hypotension, use of indomethacin, steroids, vasopressors, formula feedings) were identified as the etiology for perforation.

\section{Case:}

A female infant was delivered at 28-week gestation to a 21-yearold Gravida 4, Para 2012, with pregnancy complicated by pregnancy-induced hypertension. She was started on Nifedipine. Magnesium sulfate was started for fetal neuroprotection and maternal seizure prophylaxis. She did receive a course of betamethasone. Fetal ultrasound revealed severe intrauterine growth restriction and intermittent absence of umbilical artery (UA) dopplers. A decision was made to proceed with a primary cesarean section given a biophysical profile of 2/8 and persistent absence of end-diastolic flow on UA dopplers. Her urine drug screen and COVID screen were negative. She was $O$ positive, HIV, and hepatitis B negative. She was rubella immune, and VRDL was non-reactive. Membranes were ruptured at delivery, and she remained afebrile.

At delivery, the infant had a weak cry. She was placed in a plastic bag on the pre-heated mattress. Nasal CPAP via T-piece was provided with $\mathrm{FiO} 2$ of $40 \%$ to maintain $\mathrm{SpO} 2$ within the range for age. She was continued on nasal CPAP and transported to the NICU. The Apgar score was 7 and 8 , at one and five minutes, respectively. In the NICU, her vital signs were stable. Head circumference was $24 \mathrm{~cm}$, weight of $740 \mathrm{~g}$, and length of $34.5 \mathrm{~cm}$. Her physical examination was unremarkable for her stage of prematurity. An umbilical venous catheter (UVC) was placed, and parental nutrition was provided. She remained NPO.

On day three of life, a percutaneous intravenous central catheter (PICC) was inserted to replace UVC. The x-ray obtained to look for the PICC placement showed pneumoperitoneum (Figure). An urgent laparotomy was carried out with loop ileostomy. The gut showed no sign of necrosis. The infant remained stable post-operatively and was extubated the next day. She is currently stable on a nasal cannula and weighs 940 grams (200 grams up the birth weight). A later reconnection is planned per the pediatric surgery team.

\section{"On day three of life, a percutaneous intravenous central catheter (PICC) was inserted to replace UVC. The x-ray obtained to look for the PICC placement showed pneumoperitoneum (Figure). An urgent laparotomy was carried out with loop ileostomy. The gut showed no sign of necrosis."}

Discussion:

As noted in the case description, the perforation was spontaneous. Since birth, the infant remained NPO; there was no history of an umbilical arterial catheter, no use of indomethacin, hydrocortisone, or vasopressors. The infant was clinically stable with neither signs of infection nor any hemodynamic instability. On laboratory evaluation, she was noted to have hypermagnesemia (Table).

\section{"In a recent study, the ratio of the mean umbilical vein magnesium level to the mean maternal serum magnesium level at the time of delivery was described as $0.94 \pm 0.15$. (6) Preterm infants because of immature hepatic and renal functions are at increased risk for prolonged hypermagnesemia."}

Hypermagnesemia has been associated with intestinal perforation. $(1,2)$ The proposed pathogenesis is slowing of gut motility resulting in ileus follow by poor perfusion and perforation. Magnesium has cholinergic effects causing hypomotility of the gut - which can be detrimental in premature infants with an immature gut. Magnesium ions can replace calcium ions, disrupting the actin/ myosin interaction - reducing contractility - therefore generating atony of intestines and fecal impaction. This reaction can increase the mesenteric arterial resistance, which reduces the mesenteric blood flow resulting in ischemia and perforation. (3) The other plausible mechanism for poor gut perfusion could be the history of intrauterine growth restriction with abnormal UA dopplers.

Antenatal magnesium use is widespread in the management of preterm labor complicated by pre-eclampsia. $(4,5)$ Magnesium ions cross the placenta rapidly, and fetal levels can increase in proportion to maternal levels. In a recent study, the ratio of the mean umbilical vein magnesium level to the mean maternal serum magnesium level at the time of delivery was described as $0.94 \pm$ 0.15 . (6) Preterm infants because of immature hepatic and renal functions are at increased risk for prolonged hypermagnesemia.

In conclusion, preterm infants born to mothers who receive intravenous magnesium are at high risk for prolonged hypermagnesemia. High serum magnesium delays the gut motility and perfusion and is a potential risk factor for spontaneous gut perforation. We suggest a close observation, frequent abdominal examination, and serial serum magnesium monitoring in these groups of infants.

\section{References:}

1. Brand JM, Greer FR. Hypermagnesemia and intestinal perforation following antacid administration in a premature infant. Pediatrics 1990; 85:121-4.

2. Golzarian J, Scott HW Jr, Richards WO. Hypermagnesemiainduced paralytic ileus. Dig Dis Sci 1994; 39:1138-42.

3. Hong, J.Y., Hong, J.Y., Choi, Y. et al. Antenatal magnesium 
Table

\begin{tabular}{|c|c|c|c|c|c|c|c|c|}
\hline \multirow[b]{2}{*}{ CHEM PROFILE } & \multicolumn{2}{|l|}{ Day 1} & \multicolumn{2}{|l|}{ Day 2} & \multicolumn{2}{|l|}{ Day 2} & \multicolumn{2}{|l|}{ Day 3} \\
\hline & & & & & & & & \\
\hline Sodium & 142 & & 145 & & 148 & $\wedge$ & 143 & \\
\hline Potassium & 5.5 & ^ & 4.2 & & 4.5 & & 5.4 & 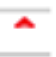 \\
\hline Chloride & 113 & 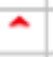 & 118 & A & 121 & $\wedge$ & 115 & $\hat{\imath}$ \\
\hline $\mathrm{CO} 2$ & 19 & $\checkmark$ & 20 & 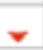 & 21 & $\checkmark$ & 21 & $\checkmark$ \\
\hline Anion Gap & 10 & & 7 & 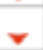 & 6 & $\gamma$ & 7 & $\checkmark$ \\
\hline BUN, Bld & 16 & & 35 & $\wedge$ & 40 & $\wedge$ & 41 & $\sim$ \\
\hline Creatinine & 0.81 & & 1.10 & & 1.00 & & 0.76 & \\
\hline eGFR if non Africa... & SEE COMMENT * & & SEE COMMENT ${ }^{*}$ & & SEE COMMENT * & & SEE COMMENT ${ }^{*}$ & \\
\hline eGFR if African Am... & SEE COMMENT & & SEE COMMENT & & SEE COMMENT & & SEE COMMENT & \\
\hline Glucose & 80 & & 71 & & 94 & & 126 & 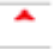 \\
\hline Calcium & 9.4 & & 9.3 & & 9.1 & & 9.4 & \\
\hline Phosphorus & 4.9 & & 4.3 & & & & 3.4 & \\
\hline Magnesium & $5.6^{x}$ & $\hat{\mathrm{A}}$ & 4.7 & a & & & 3.7 & 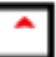 \\
\hline Alkaline Phosphatase & $\overline{214}$ & & 206 & & 759 & & 175 & \\
\hline PROTEIN TOTAL & 5.1 & $\checkmark$ & 5.2 & $\checkmark$ & 4.5 & $\checkmark$ & 4.7 & $\sim$ \\
\hline Albumin & 2.7 & & 2.9 & & 2.7 & $\checkmark$ & 2.6 & $v$ \\
\hline BILIRUBIN TOTAL & $4.0^{x}$ & & $7.8^{x}$ & & $7.2^{x}$ & & $6.0^{\circ}$ & \\
\hline Bilirubin, Direct & 0.2 & & & & & & & \\
\hline AST & 51 & $\Lambda$ & 27 & & 31 & & 40 & \\
\hline ALT & 8 & $\checkmark$ & 9 & $\checkmark$ & $<6$ & $\checkmark$ & 6 & $r$ \\
\hline
\end{tabular}

Table:

Tabulated chart showing serial serum electrolytes levels.

Serum magnesium levels are marked by a bold black line.

NEONATOLOGY TODAY is interested in publishing manuscripts from Neonatologists, Fellows, NNPs and those involved in caring for neonates on case studies, research results, hospital news, meeting announcements, and other pertinent topics.

Please submit your manuscript to: LomaLindaPublishingCompany@gmail.com

The only worldwide monthly publication
exclusively serving Pediatric and Adult
Cardiologists that focus on Congenital/
Structural Heart Disease (CHD), and
CONGENITAL
CARDOLOGY
TODAY

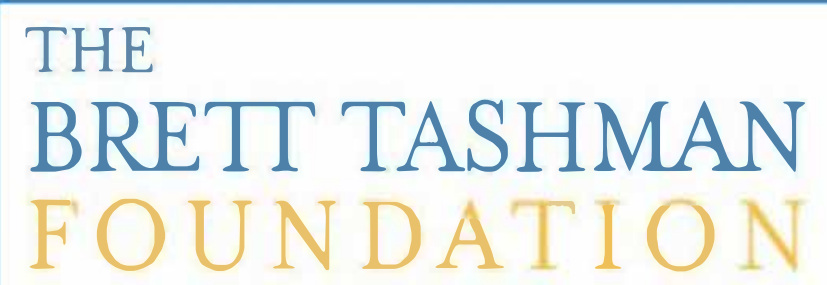

The Brett Tashman Foundation is a 501@(3) public charity. The mission of the Foundation is to find a cure for Desmoplastic Small Cell Round Tumors (DSRCT). DSRCT is an aggressive pediatric cancer for which there is no cure and no standard treatment. 100 percent of your gift will be used for research. There is no paid staff. To make your gift or for more information, go to "TheBrettTashmanFoundation.org" or phone (909) 981-1530. 


\section{Figure}
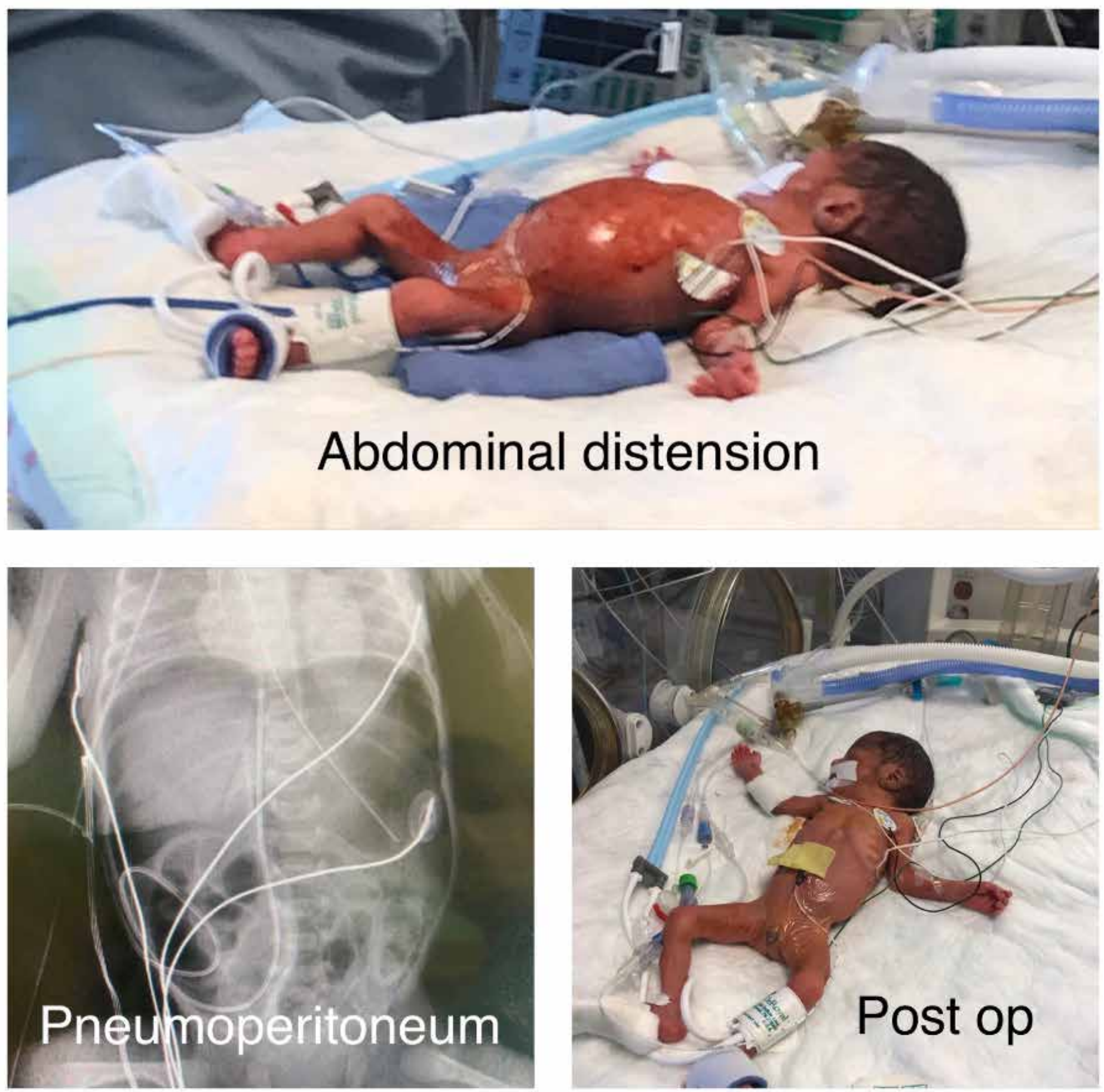

Figure:

Top panel: Pre-operative picture showing distended abdomen.

Bottom Right panel: Post-operative picture showing flat abdomen.

Bottom Left panel: X-ray showing the football sign (free air in the peritoneum). 
sulfate treatment and risk of necrotizing enterocolitis in preterm infants born at less than 32 weeks of gestation. Sci Rep 10, 12826 (2020). https://doi.org/10.1038/s41598-02069785-3

4. Mittendorf $R$, Pryde PGA review of the role for magnesium sulphate in preterm labour. BJOG. 2005; 112: 84-88

5. Duley L, Gulmezoglu A.M, Henderson-Smart D.J, Chou D. Magnesium sulphate and other anticonvulsants for women with pre-eclampsia. Cochrane Database Syst Rev. 2010: CD000025

6. Brookfield KF, Su F, Elkomy MH, et al. Pharmacokinetics and placental transfer of magnesium sulfate in pregnant women. Am J Obstet Gynecol 2016; 214:737.e1-9.

Disclosure: The authors report no conflicts of interest.

NT

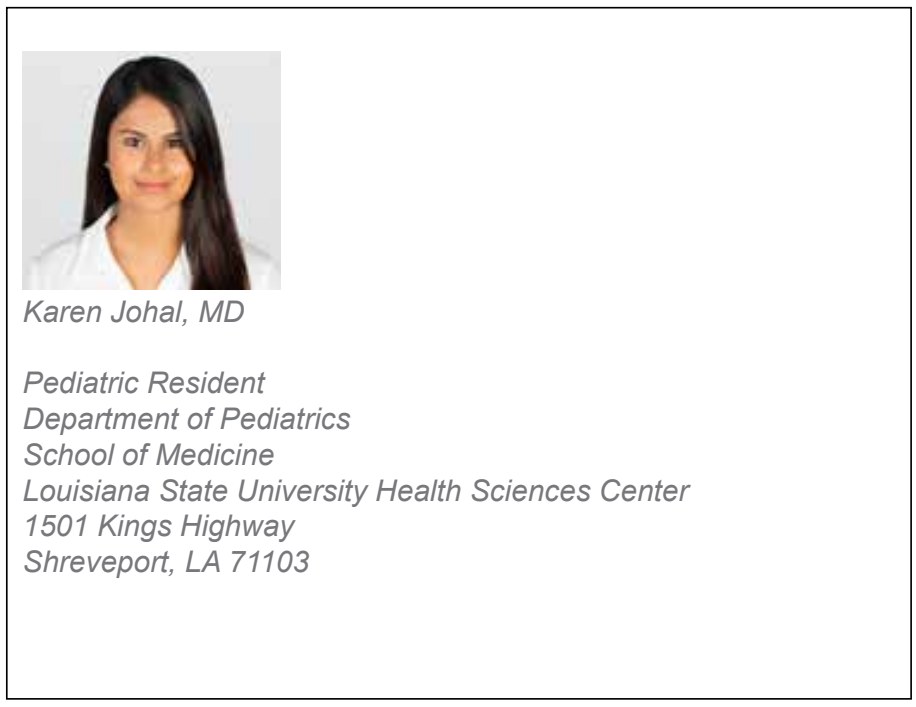

Corresponding Author
Shabih Manzar, MD
Attending
Department of Pediatrics
Division of Neonatology
College of Medicine
Louisiana State University of Health Sciences
1501 Kings Highway
Shreveport, LA 71130
Telephone: 318-626- 4374
Fax: 318-698-4305
Email: smanza@/suhsc.edu
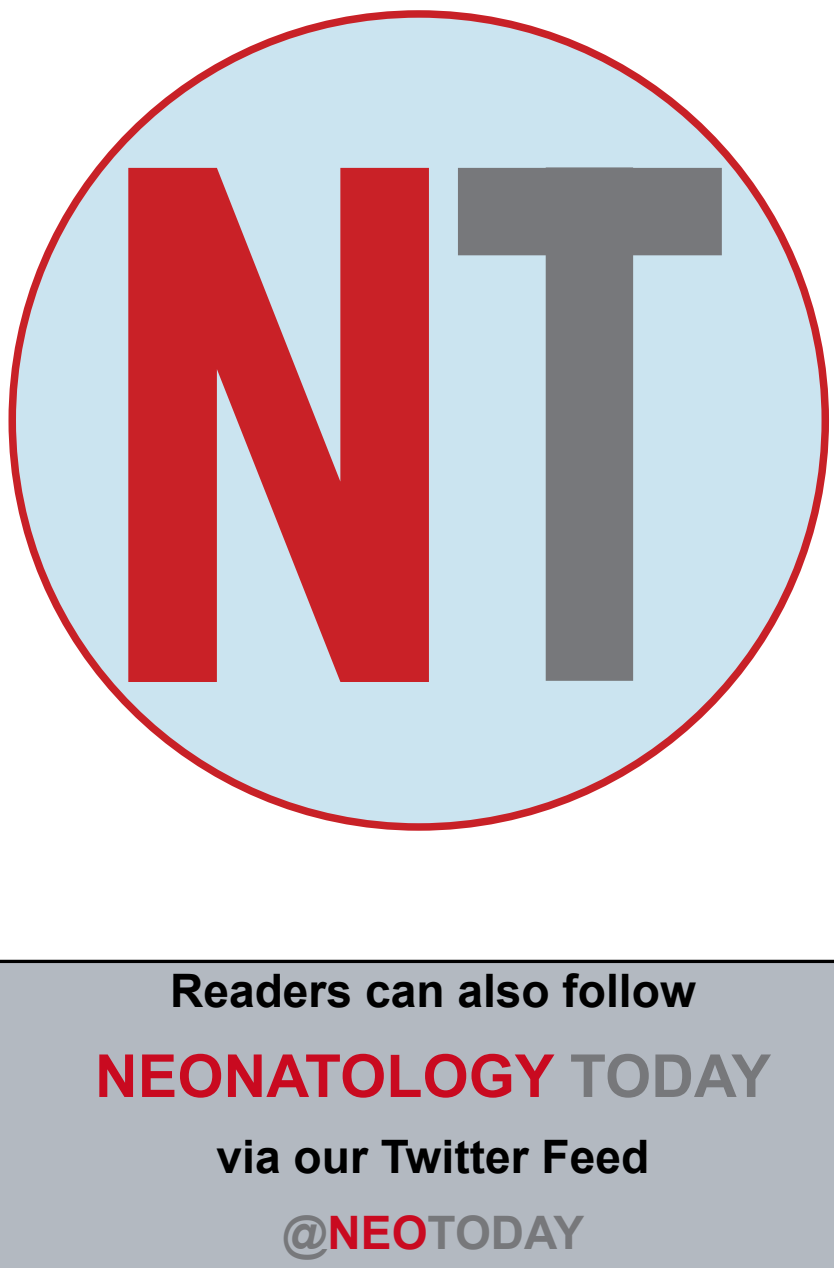

NATIONAL PERINATAL ASSOCIATION

CORONAVIRUS

COVID-19

RELIABLE

RESOURCES:

- CDC: 2019 Novel Coronavirus

- The Lancet: CoviD-19 and pregnancy

- MotherToBaby: coronaviruses

- WHO: Emerging respiratory viruses

STAY INFORMED.

ONational Perinatal 